Enferm Bras 2020;19(3):220-9

https://doi.org/10.33233/eb.v19i3.3254

\title{
ARTIGO ORIGINAL \\ Crenças de Rokeach segundo idosos: análise hierárquica a luz das teorias do envelhecimento humano
}

Laércio Deleon de Melo, M.Sc. ${ }^{*}$, Cristina Arreguy-Sena, D.Sc. ${ }^{* *}$, Antônio Marcos Tosoli Gomes, D.Sc. ${ }^{* *}$, Paulo Ferreira Pinto, D.Sc. ${ }^{* * *}$, Girlene Alves da Silva, D.Sc. ${ }^{* * * *}$, Pedro Miguel Dinis Parreira, D.Sc. ${ }^{* \star * * *}$

${ }^{\star}$ Enfermeiro, Universidade Federal de Juiz de Fora (UFJF), Juiz de Fora/MG, Brasil, ${ }^{*}$ Enfermeira, Universidade Federal de Juiz de Fora (UFJF), Professora Aposentada, Juiz de Fora/MG, Brasil, ${ }^{* * \star}$ Enfermeiro, Professor Adjunto, Universidade Estadual do Rio de Janeiro (UERJ), Rio de Janeiro/RJ, Brasil, ${ }^{* * *}$ Educador Físico, Universidade Federal de Juiz de Fora (UFJF), Professor Associado, Juiz de Fora/MG, ${ }^{* * \star * \star}$ Enfermeira, Professora Adjunta, Universidade Federal de Juiz de Fora (UFJF), Juiz de Fora/MG, ${ }^{* * * * *}$ Professor Adjunto, Escola Superior de Enfermagem de Coimbra, Lisboa, Portugal

Recebido em 27 de setembro de 2019; aceito em 13 de março de 2020.

Correspondência: Laércio Deleon de Melo, Rua Doutor Dirceu de Andrade, 201/305 Dom Bosco 36025-330 Juiz de Fora MG

Laércio Deleon de Melo: laerciodl28@hotmail.com

Cristina Arreguy-Sena: cristina.arreguy@gmail.com

Antônio Marcos Tosoli Gomes: mtosoli@gmail.com

Paulo Ferreira Pinto: paulo.ferpinto@gmail.com

Girlene Alves da Silva: girlenealves.silva@ufjf.edu.br.

Pedro Miguel Dinis Parreira: pedromiguel.parreira@gmail.com

\section{Resumo}

Introdução: As compreensões sobre o envelhecimento estão subordinadas a variáveis individuais e coletivas, nos contextos: biopsicossocial, espiritual, político e cultural; fato capaz de justificar os diferentes modos de se estabelecer crenças e formas explicativas peculiares a um grupo sobre o envelhecer. Objetivo: Analisar as crenças de idosos sobre o processo de envelhecimento segundo o sistema hierárquico no eixo de centralidade/perifericidade e sua aproximação com as teorias do envelhecimento humano. Métodos: Pesquisa exploratória com abordagem qualitativa realizada com 93 pessoas idosas adstritas à Atenção Primária à Saúde (APS) cujas crenças foram categorizadas segundo Rokeach e teorias do envelhecimento humano. Realizadas entrevistas individuais em domicílio, com gravação de áudio, desencadeadas por questões norteadoras. Tratamento de dados em softwares NVivo Pro11 e SPSS versão 24 com análise de conteúdo. Atendidos todos os requisitos éticos/legais. Resultados: Obtidas 696 crenças dos tipos $\mathrm{A}$ e $\mathrm{B}, 163$ tipo $\mathrm{C}$ e 1.006 dos tipos $\mathrm{D}$ e $\mathrm{E}$. O sistema de crenças tendeu à perifericidade, explicitando os núcleos temáticos de argumentações utilizadas pelos participantes. Conclusão: Identificadas crenças modificáveis de caráter negativo sobre o envelhecimento, que possuíam explicações delineadas nas teorias do envelhecimento. Ressalta-se a necessidade de empreender esforços para revertê-las e realinhá-las ao equilíbrio do eixo de centralidade/perifericidade.

Palavras-chave: Enfermagem geriátrica, envelhecimento, saúde do idoso, psicologia social.

\footnotetext{
Abstract

Rokeach beliefs according to elderly: hierarchical analysis in the light of human aging theories

Introduction: Understanding about aging is subordinated to individual and collective variables, in the following contexts: biopsychosocial, spiritual, political and cultural; fact capable of justifying the different ways of establishing beliefs and explanatory forms peculiar to a group about aging. Objective: To analyse the beliefs of the elderly about the aging process according to the hierarchical system in the centrality/periphery axis and its approximation with the theories of
} 
human aging. Methods: Exploratory research with qualitative approach conducted with 93 elderly people enrolled in Primary Health Care whose beliefs were categorized according to Rokeach and theories of human aging. Individual interviews were conducted at home, with audio recording, triggered by guiding questions. Data processing in NVivo Pro11 and SPSS version 24 software with content analysis. Met all ethical/legal requirements. Results: 696 types A and B beliefs, 163 type $C$ and 1,006 types $D$ and $E$ beliefs were obtained. The belief system tended to be peripheral, explaining the thematic core of arguments used by the participants. Conclusion: Modifiable negative beliefs about aging were identified, which had explanations outlined in aging theories. We emphasize the need to make efforts to reverse them and realign them to the balance of the axis of centrality/periphery.

Keywords: Geriatric nursing, aging, health of elderly, psychology, social.

\section{Resumen \\ Creencias de Rokeach según el anciano: análisis jerárquico a la luz de las teorías del envejecimiento humano}

Introducción: La comprensión sobre el envejecimiento está subordinada a variables individuales y colectivas, en los siguientes contextos: biopsicosocial, espiritual, político y cultural; hecho capaz de justificar las diferentes formas de establecer creencias y formas explicativas propias de un grupo sobre el envejecimiento. Objetivo: Analizar las creencias de las personas mayores sobre el proceso de envejecimiento según el sistema jerárquico en el eje centralidad/periferia y su aproximación a las teorías del envejecimiento humano. Métodos: Investigación exploratoria con enfoque cualitativo realizada con 93 personas mayores inscritas en Atención Primaria de Salud cuyas creencias se clasificaron de acuerdo con Rokeach y las teorías del envejecimiento humano. Las entrevistas individuales se realizaron en casa, con grabación de audio, desencadenada por preguntas orientadoras. Procesamiento de datos en el software NVivo Pro11 y SPSS versión 24 con análisis de contenido. Cumplió con todos los requisitos éticos/legales. Resultados: Se obtuvieron 696 creencias tipo A y B, 163 creencias tipo C y 1.006 tipos D y E. El sistema de creencias tendió a ser periférico, explicando el núcleo temático de los argumentos utilizados por los participantes. Conclusión: Se identificaron creencias negativas modificables sobre el envejecimiento, que tenían explicaciones esbozadas en las teorías del envejecimiento. Hacemos hincapié en la necesidad de hacer esfuerzos para revertirlos y realinearlos al equilibrio del eje de centralidad/periferia.

Palabras-clave: Enfermería geriátrica, envejecimiento, salud del anciano, psicología social.

\section{Introdução}

O processo de envelhecimento é reconhecido mundialmente como um evento complexo e multifatorial [1,2], que possui como variáveis transversais o gênero e a cultura [3]. O mesmo cursa com manifestações clínicas diversas, capazes de repercutir sobre a autonomia e independência do idoso, de forma linear e progressiva, caracterizando as síndromes geriátricas, cujas características são comuns ao grupo que envelhece [4,5].

As projeções estatísticas, no contexto global, indicam um crescimento de $56 \%$ para pessoas com idade $\geq 60$ anos, podendo atingir 1,4 bilhão em 2030 e 2,1 bilhões em 2050. Destes, as oldest-old, pessoas com idade $\geq 80$ anos, podem representar 434 milhões [1-2]. No Brasil, as pessoas $\geq 65$ anos serão aproximadamente 58,4 milhões ( $26,7 \%$ da população) em 2060 [5].

As compreensões sobre o envelhecimento estão subordinadas a variáveis individuais e coletivas, nos contextos: biopsicossocial, espiritual, político e cultural; fato capaz de justificar os diferentes modos de se estabelecer crenças e formas explicativas peculiares a um grupo sobre o envelhecer, podendo adotar diferentes paradigmas [2,6,7].

Para captar as crenças do grupo investigado, optou-se pelo sistema explicativo proposto por Rokeach. Para ele, crença é uma inferência feita pelo observador sobre estados e expectativas básicas que possibilita acessar ideias, conceitos, convicções e atitudes adotadas pelas pessoas mediante um fato, correlacionando-os a fatores que influenciam o cotidiano, a autoimagem e o processo de saúde-doença [8,9].

Aliar a estrutura de crenças com as correntes teórico-explicativas para o processo de envelhecimento humano nas abordagens: biológica, psicológica, sociológica e epidemiológica; proporciona respaldo e compreensão aprofundada a respeito da origem das crenças, conforme cada perspectiva teórica identificada nos discursos advindos do grupo [8].

No ponto de vista da atuação do enfermeiro, esta investigação se justifica pela necessidade de explicitar quais são as possíveis respostas que emergem deste grupo sobre 0 
processo de envelhecimento, as repercussões sobre o seu cotidiano, hábitos e concepções (autoimagem, autopercepção, pontos facilitadores/dificultadores e crenças compartilhadas). Estas respostas favorecem a compreensão de como os idosos enfrentam essa etapa da vida e possibilitam a identificação de demandas por cuidados de Enfermagem, o que pode contribuir para seu redimensionamento e planejamento.

A lacuna científica existente baseia-se na necessidade de se correlacionar as crenças de Rokeach com as teorias explicativas do processo de envelhecimento humano segundo a ótica de idosos. Diante do exposto, objetivou- analisar as crenças de idosos sobre o processo de envelhecimento segundo o sistema hierárquico no eixo de centralidade/perifericidade e sua aproximação com as teorias do envelhecimento humano.

\section{Material e métodos}

Pesquisa qualitativa exploratória, estruturada a partir do sistema de crenças de Rokeach e formas explicativas do processo de envelhecimento. Foi realizada em área adstrita a uma Unidade Básica de Saúde (UBS) em Minas Gerais, Brasil, e os participantes foram abordados em domicílio, após recrutamento realizado por convite individual.

Foram elegíveis pessoas com idade $\geq 65$ anos que apresentavam nível de consciência compatível com a abordagem qualitativa de produção de discurso gravado. Foram excluídas, durante a coleta: 16 por desistência e uma por mudança de endereço. A amostra por inquérito se compôs de 93 idosos advindos de uma investigação matriz $(\mathrm{N}=110)$.

A determinação da idade de 60 ou 65 anos para que uma pessoa seja considerada idosa pode não corresponder à realidade quando se avaliam culturas distintas, o que implica considerála complexa e multifatorial, uma vez que envolve condições de vida, trabalho, lazer, expectativa de vida e investimentos em hábitos saudáveis ao longo da vida, para o envelhecimento bemsucedido $[1,8]$. Nesta perspectiva, optou-se pelo delineamento de pessoas com idade $\geq 65$ anos para serem inclusas nesta investigação.

O instrumento de coleta de dados foi estruturado em: caracterização sociodemográfica e entrevista individual em profundidade gravada a partir das questões norteadoras: a) conte-me um caso a respeito do cotidiano de pessoas idosas; b) diga-me como soube do mesmo e o quanto o considera característico de uma pessoa que está envelhecendo.

Processo de coleta de dados compreendido entre janeiro e março 2015, em abordagem única, por um único pesquisador, visando reduzir viés de abordagem e compatibilizar o tempo com a disponibilidade dos participantes. Coleta de dados com duração $\geq 60$ minutos.

Os dados sociodemográficos foram consolidados em software Statistical Package for the Social Sciences (SPSS) versão 24 e os da entrevista gravada no NVivo Pro11®.

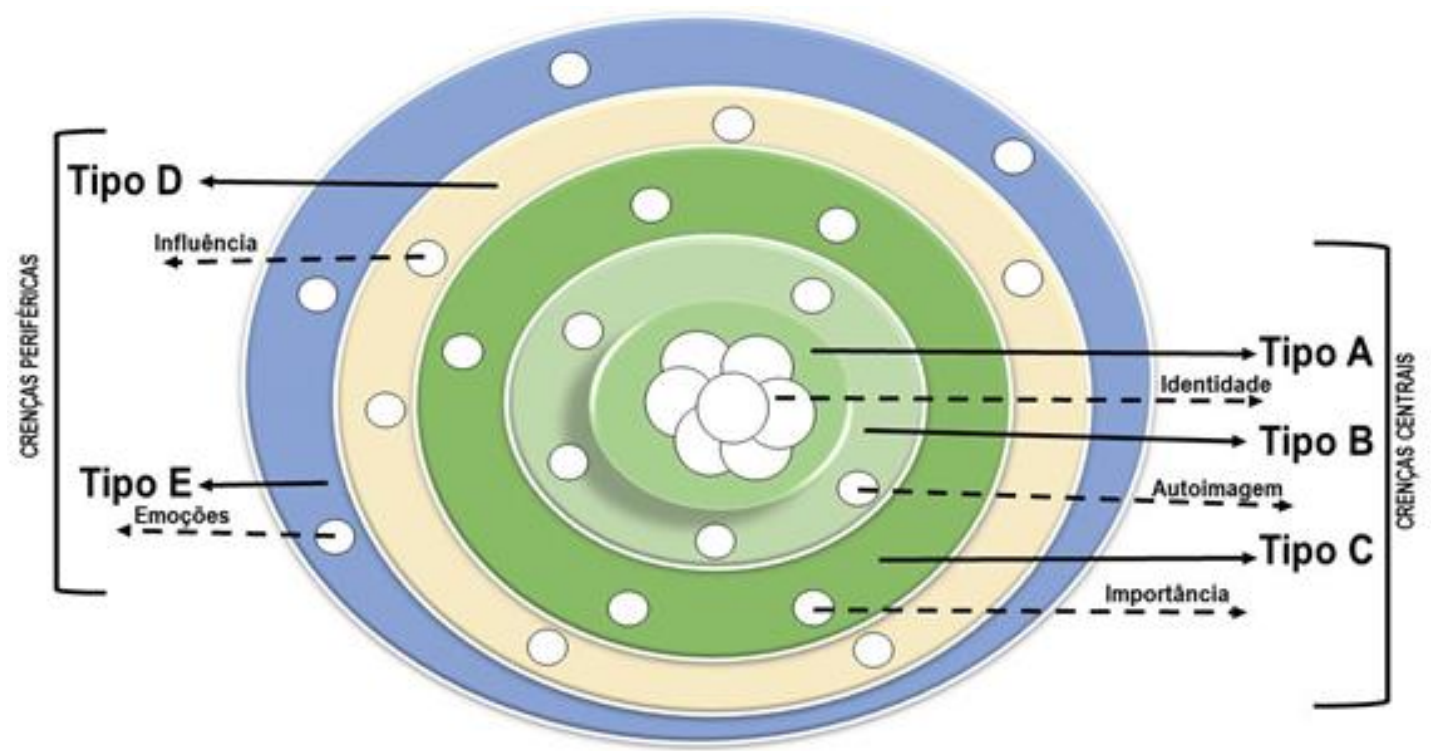

Fonte: Barcelos 2007 p.118 [12]; adaptado pelos autores.

Figura 1 - Esquema representativo do eixo de centralidade/perifericidade das crenças de Rokeach, usando analogia à estrutura de um átomo. 
Realizada análise de conteúdo segundo etapas: pré-análise; exploração do material (codificação) e tratamento dos resultados obtidos (interpretação) [10] dos quais os conteúdos foram alocados em nós (critério de similaridade). Adensamento teórico confirmado (coeficiente de Jaccard $\geq 0,70)[8]$.

Os discursos foram categorizados segundo os tipos de crenças de Rokeach, cujos tipos de crenças alocaram-se imaginariamente num eixo de centralidade/perifericidade (Figura 1) $[9,11,12]$.

A análise foi realizada de forma comparativa à estrutura de um átomo, no qual os prótons correspondem às crenças centrais $(\mathrm{A}$ e $\mathrm{B})$, no eixo imaginário, devido à sua estabilidade, e os elétrons compostos pelas demais crenças $(C, D$ e E), que são potencialmente influenciáveis $e$ modificáveis conforme for ampliado o sistema crenças, o vivenciar e as diferentes formas de compreensão do grupo, correspondendo ao eixo periférico. As crenças do tipo A (consenso unânime) são compartilhadas por todos e raramente são alvo de discussão ou caem em controvérsia; as crenças de consenso zero (tipo B) são mantidas em sigilo e por isso são resistentes ao processo de mudança. O sistema periférico é composto pelas crenças do tipo $C$ que normalmente são submetidas a uma autoridade conferida ou atribuídas a alguém; derivadas (tipo D), oriundas de meios de influências; e as do tipo $E$ (inconsequentes) são referentes a situações de preferência ou gosto, apresentando diferentes arbitrariedades [9,11].

A compreensão/análise dos alicerces das percepções de idosos baseou-se nas correntes teórico-explicativas do envelhecimento (Figura 2) [7,8]. As explicações biológicas referem-se aos fatores genéticos como determinantes do envelhecimento contidos nas perspectivas genéticas e à perda de funcionalidade causada pelo acúmulo aleatório de lesões por ações ambientais, como justificativa ao declínio fisiológico progressivo, fundamentado nas abordagens estocásticas [7,13]. As dimensões psicológicas buscam estabelecer explicações sobre as influências do envelhecimento nos pensamentos e comportamentos humanos $[7,14]$.
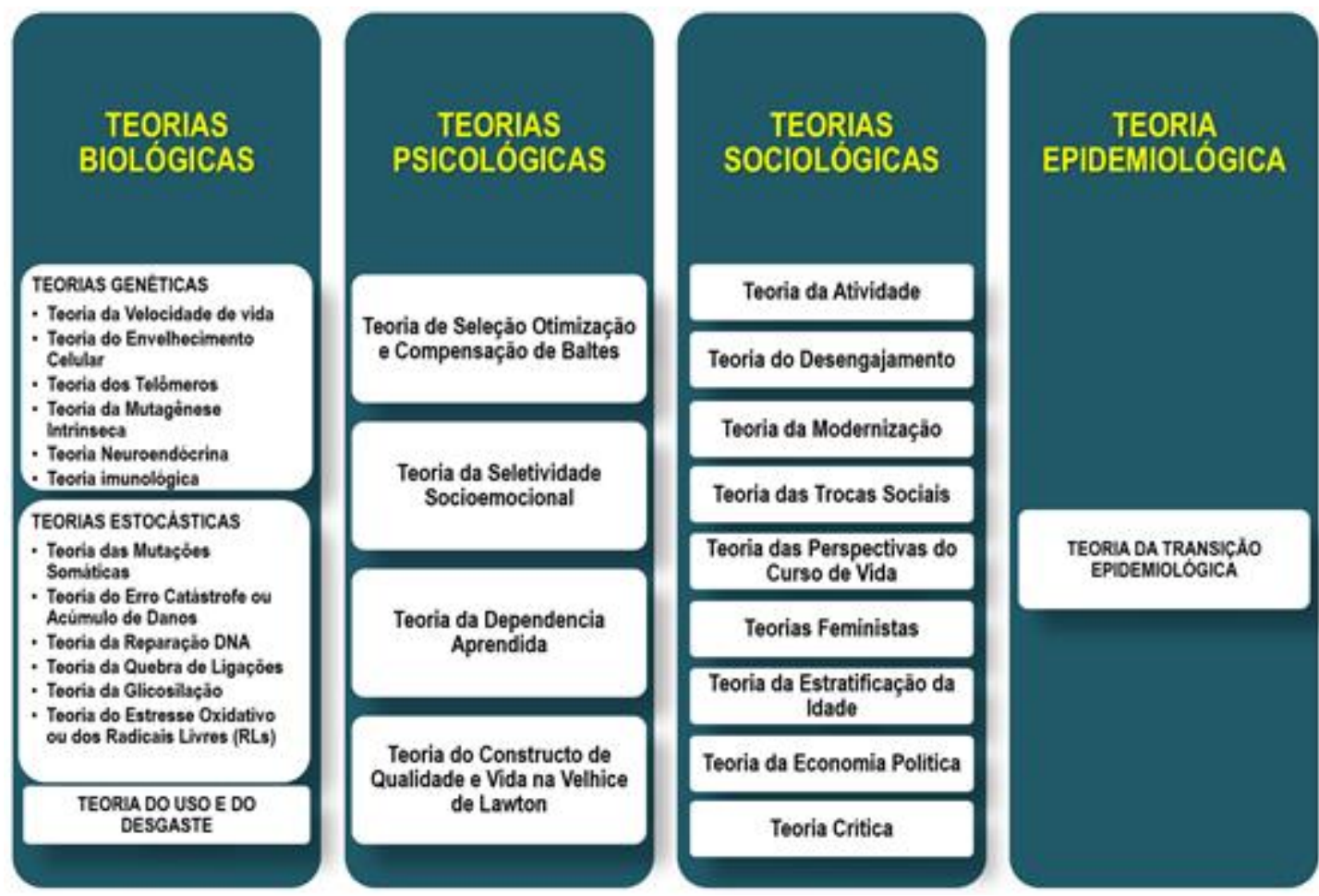

Fonte: os autores.

Figura 2 - Esquema das concepções teórico-explicativas do envelhecimento humano.

As perspectivas sociológicas descrevem o engajamento social de idosos e as relações estabelecidas socialmente $[7,15]$. As reflexões sobre o aumento da expectativa de vida e o quantitativo de idosos no mundo estão ancoradas na teoria epidemiológica [8].

Os conteúdos categóricos sobre como as pessoas idosas perceberam o processo de envelhecimento foi aproximado das formas teóricas reificadas que explicam como 0 envelhecimento humano é retratado na perspectiva do conhecimento do grupo. Esse conteúdo foi aproximado das formas explicativas existentes no sistema de crenças de Rokeach, sendo 
utilizada a técnica analítica dedutiva-indutiva que permitiu identificar o quanto o sistema de crenças emergente era estável (central) ou instável (periférica) $[9,16,17]$.

Foram atendidos todos os requisitos ético-legais de pesquisa em humanos, conforme legislação (inter)nacional, sendo a investigação matriz aprovada (Parecer consubstanciado $\mathrm{n}$. 341.116 aprovado em 11/07/2013). Cabe mencionar o respeito ao anonimato dos participantes e a aquiescência de sua participação mediante assinatura do Termo de Consentimento Livre e Esclarecido (TCLE) pós-informado.

\section{Resultados e discussão}

A maioria dos 93 idosos eram mulheres (58 - 62,4\%); com idade média de 72 anos (variabilidade: 65 a 96); cor de pele autodeclarada parda (48 - 51,6\%); com companheiro (50 $53,76 \%$ ); média de quatro filhos (variabilidade: 1 a 21 ) e tinham até oito anos de escolaridade (82 - 88,15\%). Atuaram em serviços domésticos $38(40,87 \%)$ e na construção civil $20(21,50 \%)$. Possuíam renda média $\leq 2$ salários mínimos 87 (94,5\%), dos quais 86 (92,46\%) eram aposentados/pensionistas com tempo médio de benefício de 9,5 anos (variância: 0 a 37).

O sistema de crenças foi composto por 1.865 emissões que contemplaram o processo de envelhecimento, cuja estrutura hierárquica possibilitou identificar que 859 crenças tenderam à nuclearidade e 1.006 à perifericidade (Figura 3).

\begin{tabular}{|c|c|c|c|c|}
\hline $\begin{array}{l}\text { Sintema } \\
\text { Crencas } \\
\text { Rokesch }\end{array}$ & $\begin{array}{c}\text { Nimero } \\
\text { crencas } \\
\text { (8) }\end{array}$ & Fragmentos de discursos dos participantes segundo tipo de crenças & 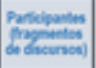 & $\begin{array}{l}\text { Dondograma o Grifico de } \\
\text { Cireullo }\end{array}$ \\
\hline 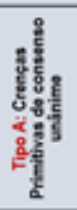 & $\begin{array}{c}362 \\
(19,4 \%)\end{array}$ & 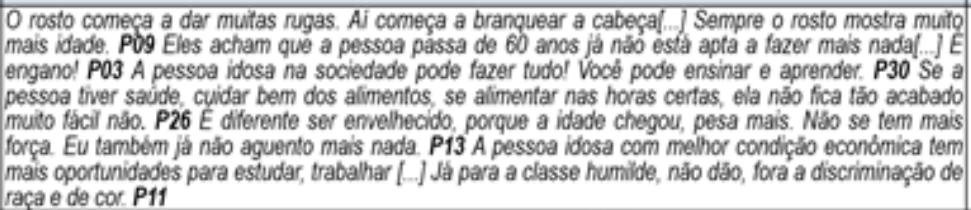 & $78(343)$ & \\
\hline 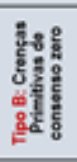 & $\begin{array}{c}334 \\
(14,9 \%)\end{array}$ & 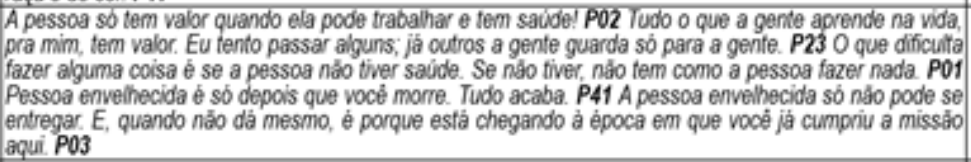 & $83(314)$ & \\
\hline 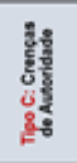 & $\begin{array}{c}163 \\
(8,7 \%)\end{array}$ & 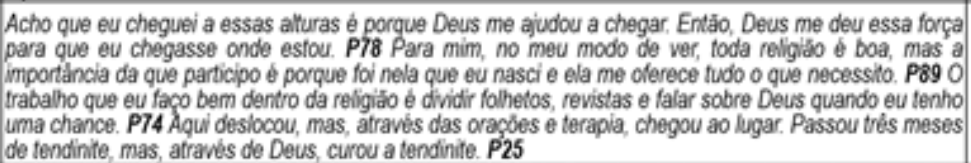 & $80(163)$ & \\
\hline 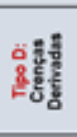 & $\begin{array}{c}722 \\
(38,7 \%)\end{array}$ & 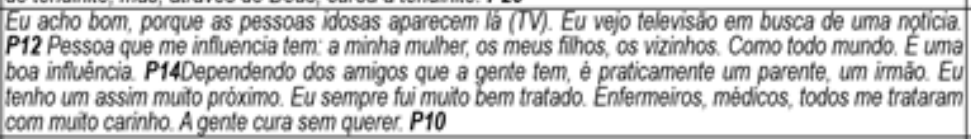 & $85(722)$ & 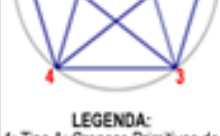 \\
\hline 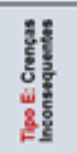 & $\begin{array}{c}284 \\
(15,2 \%)\end{array}$ & $\begin{array}{l}\text { Eu gosto de trabalhar muito! Eu nāo paro nem um minuto. Eu faço colchas de retalhos e bordo, planto } \\
\text { fiorés. P19 Eu preferia antes, quando eu era bem atendido pelo enfermeiro, médico e tudo, mas agora nâo. } \\
\text { P33 Eu finha boa convivéncia la no Programa X[ ] Eu ia para me divertit, encontrar com as amigas. P43 }\end{array}$ & $82(284)$ & 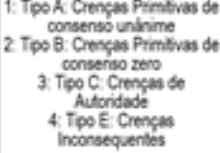 \\
\hline
\end{tabular}

Fonte: programa Nvivo Pro $11 \AA$.

Figura 3 - Sistema de Crenças de Rokeack segundo o tpo de crenças e exemplificação segundo fragmentos de discursos.

A saturação dos dados apresentada para os tipos de crenças e teorias explicativas foi corroborada por dendograma e gráfico de círculo (Figura 4). Foi possível alocar hierarquicamente os tipos de crenças, compreender seus conteúdos emergentes e identificar sua conectividade com as teorias explicativas do processo de envelhecimento humano e o continuum saúde/doença.

Como conteúdo das crenças do Tipo A (consenso unânime), emergiram: 1) caracterização do processo de agrisalhamento; 2) sentimento de ser útil; 3) inserção em atividades; 4) hábitos de vida e; 5) limitações.

O discurso do P09 descreveu sinais do processo de agrisalhamento identificados na aparência como caracterização desta etapa da vida. Sua crença está fundamentada na teoria do envelhecimento celular ao descrever a caracterização física e visual do processo de envelhecimento, cujas manifestações envolvem: rugas, cabelos brancos, alterações sobre a postura física e na marcha $[7,13]$. 
O sentimento de ser útil foi vinculado às percepções quanto a pouca valorização do idoso pelas pessoas que convive, pelo menos não correspondente à intensidade de que eles se julgam merecedores. No convívio social, eles se interessam por provarem que ainda possuem utilidade e aptidão na realização de algumas atividades. Esta concepção pode ser explicada pela Teoria da Atividade, que defende que, apesar das perdas físicas, psicológicas e sociais, os vínculos sociais e a autonomia persistem visando a manutenção da participação social, a satisfação pessoal e autoconceito de aspectos positivos sobre o envelhecimento $[7,15]$.

De modo corroborativo a existência de crenças que demonstraram uma potencialidade do idoso em se inserir em atividades laborais ou voluntárias de modo a se manterem ativos, autônomos e independentes. A aproximação teórica descrita envolve a Teoria da Perspectiva do Curso de Vida, ao retratar o interesse pela integração ao meio social no compartilhamento de conhecimentos e experiências de vida com o grupo $[8,15]$.

As categorias definidas a priori (segundo referenciais teóricos), justifica a ausência de alguns conteúdos ou presença insuficiente para gerar correlação no gráfico de círculo.

\begin{tabular}{|c|c|c|c|c|c|}
\hline \multicolumn{3}{|r|}{ Referencial } & \multirow{2}{*}{$\begin{array}{c}\text { Fonte } \\
9\end{array}$} & \multirow{2}{*}{$\begin{array}{c}\text { Referências } \\
9\end{array}$} & Gráfico de Circulo \\
\hline \multirow{13}{*}{ 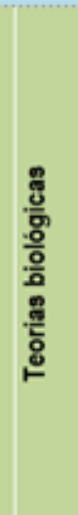 } & \multirow{6}{*}{ 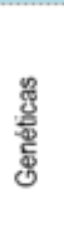 } & 1.Velocidade de vida & & & \\
\hline & & 2. Envelhecimento celular & 40 & 52 & \\
\hline & & 3. Mutagênese intrínseca & 2 & 2 & \\
\hline & & 4. Neuroendócrina & 10 & 11 & \\
\hline & & 5. Imunológica & 15 & 16 & \\
\hline & & 6. Telômeros & $\cdot$ & - & \\
\hline & \multirow{6}{*}{ 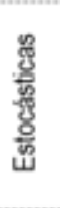 } & 1. Mutações somáticas & 17 & 21 & \\
\hline & & 2. Radicais livres & 22 & 32 & \\
\hline & & 3. Reparação do DNA & 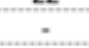 & $\therefore$ & \\
\hline & & 4. Erro catástrofe ou acúmulo de danos & 1 & 1 & \\
\hline & & 5. Quebra de ligaçōes & $\therefore$ & $\therefore$ & \\
\hline & & 6. Glicosilação & - & - & \\
\hline & \multicolumn{2}{|c|}{ Uso e desgaste } & 57 & 103 & \\
\hline \multirow{4}{*}{ 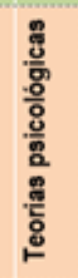 } & \multicolumn{2}{|c|}{ 1. Seleção, otimização e compensação de Baltes } & 41 & 75 & \\
\hline & \multicolumn{2}{|c|}{ 2. Seletividade socioemocional } & 66 & 104 & \\
\hline & \multicolumn{2}{|c|}{ 3.Dependéncia aprendida } & 51 & 90 & \\
\hline & \multicolumn{2}{|r|}{ 4. Constructo de qualidade de vida na velhice de Lawton } & 84 & 306 & \\
\hline \multirow{10}{*}{ 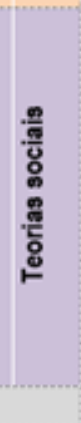 } & \multicolumn{2}{|c|}{ 1. Atividade } & 77 & 224 & \\
\hline & \multicolumn{2}{|c|}{ 2. Desengajamento } & 63 & 130 & \\
\hline & \multicolumn{2}{|c|}{ 3. Modernização } & 27 & 47 & \\
\hline & \multicolumn{2}{|c|}{ 4. Trocas sociais } & 84 & 376 & \\
\hline & \multicolumn{2}{|c|}{ 5. Perspectiva do curso de vida } & 30 & 43 & \\
\hline & \multicolumn{2}{|c|}{ 6. Feministas } & 3 & 3 & \\
\hline & \multicolumn{2}{|c|}{ 7. Estratificação da idade } & 53 & 65 & \\
\hline & \multicolumn{2}{|c|}{ 8. Economia politica } & 85 & 316 & \\
\hline & \multicolumn{2}{|c|}{ 9. Critica } & 72 & 191 & \\
\hline & \multicolumn{2}{|c|}{ Transiçăo epidemiológica } & 3 & 3 & \\
\hline \multirow{5}{*}{ 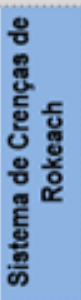 } & \multicolumn{2}{|c|}{ 1. Tipo $\mathrm{A}$ - consenso unânime $100 \%$} & 78 & 343 & \\
\hline & \multicolumn{2}{|c|}{ 2. Tipo B - consenso zero } & 83 & 314 & \\
\hline & \multicolumn{2}{|c|}{ 3. Tipo C - autoridade } & 80 & 163 & \\
\hline & \multicolumn{2}{|c|}{ 4. Tipo D-derivadas } & 85 & 722 & \\
\hline & \multicolumn{2}{|c|}{ 5. Tipo E - inconsequentes } & 82 & 284 & \\
\hline
\end{tabular}

Fonte: programa Nvivo Pro $11 \AA$.

Figura 4 - Critérios de saturação dos dados, segundo forças de correlações entre categorias expressas pelo gráfico de círculo. 
As crenças a respeito dos hábitos de vida contemplaram: alimentação, prática de atividade física e qualidade do sono/repouso, como determinantes do envelhecimento saudável, ativo e bem-sucedido, conforme discurso do P26. A possível aproximação teórica envolve a Teoria de Seleção, Otimização e Compensação de Baltes, a partir de reflexões do idoso sobre o manejo efetivo do seu processo de envelhecimento, o que justifica sua inserção em hábitos de vida saudáveis em busca pelo envelhecimento bem-sucedido $[7,14]$.

As crenças de que o idoso é aquele que "não pode fazer as coisas", justificados pela idade, fraqueza e indisposição. As explicativas teóricas perpassam as dimensões biológicas (resistência e força), psicológicas (motivação e dedicação) e sociais (desengajamento) de modo a descreverem possíveis limitações progressivas/características da senilidade [12-15].

O conteúdo das crenças do tipo $B$ (Consenso Zero) não depende do respaldo das demais pessoas ou do grupo para a sua reafirmação e/ou manutenção, não possuem referência externa ou própria em que possa ser influenciada [9-11]. Foram categorizadas em: 1) valores; 2) aprendizado de vida; 3) continuum saúde/doença; 4) avaliação de ser idoso e 5) finitude.

Os valores foram vinculados à capacidade do idoso em manter-se produtivo, trabalhar, ter saúde, algo contrário à inutilidade. $O$ aprendizado estruturado a partir das experiências vividas foi considerado outra virtude a ponto de compartilhar experiências com o grupo baseados em valores ético-morais e distinções pessoais entre o certo e o errado (Figura 3). A explicativa teórica envolve o Constructo de Qualidade de Vida na Velhice de Lawton, que descreve percepções do idoso a respeito de suas relações no contexto social e remete à avaliação multidimensional vinculada a critérios intrapessoais e sócio-normativos [8,14].

As crenças associadas ao continuum saúde/doença descreveram problemas de saúde que o idoso vivencia somadas as complicações/limitações possíveis como: redução de força física, presença de dor e dificuldades na locomoção, conforme discurso do P01. Cabe mencionar que envelhecimento, quando acompanhado pelo aumento das cargas de doenças e medicalização, requer que o idoso seja capaz de enfrentar/conviver com as modificações de forma a manter-se ativo, participativo e inserido socialmente, apesar das limitações [1,3], a exemplo da polifarmácia (critério classificatório de vulnerabilidade) $[4,5]$.

A avaliação de ser idoso quanto a elementos negativos do envelhecer apresentou crenças relacionadas à: morte, ausência de saúde e/ou deficiência física, limitações na comunicação ou inserção em atividades conforme expresso pelo P41. A Teoria do Uso e do Desgaste pode oferecer respaldo às percepções apresentadas ao abordá-las como resultante do acúmulo de agressões ambientais cotidianas que diminuem a capacidade do organismo em recuperar-se de forma efetiva. Dessa forma, ferimentos, infecções e inflamações somam-se ao longo dos anos, ocasionando alterações celulares, teciduais e orgânicos na intensificação do processo de envelhecimento $[7,13]$.

A finitude foi vinculada à concepção do envelhecimento como uma etapa final da vida. Ela remete à dicotomia entre lutar pela vida ou entregar-se as limitações de modo a aceitar/aguardar a morte. Visto a aproximação da morte, esta pode assumir um protagonismo mediante a desistência do idoso em viver. A aproximação teórica envolvida é a da Seletividade Socioemocional ao possibilitar a compreensão do idoso como capaz de reconhecer sua finitude e alterar seus comportamentos e significação de suas relações com o meio social e familiar, baseado em suas emoções $[8,14]$.

As crenças do tipo $C$ (Autoridade) possuem flexibilidade indispensável para se reordenarem, podendo amortecer e acomodar o sistema e por vezes serem consideradas centrais ou periféricas [9-10]. Elas foram relacionadas à: 1) Deus; 2) religiosidade/espiritualidade; 3) prática religiosa; 4) posicionamento religioso e busca pelos serviços de saúde.

A crença em Deus foi à autoridade máxima sobre a vida do idoso, capaz de influenciá-lo e direcionar suas decisões, remetendo a sensação de bem-estar, proteção pessoal, renovação de energias e motivações pessoais, conforme fala do P78. De forma relacionada à religiosidade/espiritualidade exprimiu a dimensão do segmento religioso em que o idoso está inserido. É utilizada para justificar sua prática religiosa, atribuindo motivos/importância no contexto vivido; sendo ainda uma forma de obtenção de conforto psicológico/espiritual (P89).

A aproximação teórica envolve a Seleção, Otimização e Compensação de Baltes, que explica a forma como o idoso pode manejar e se adaptar às diferentes mudanças [7,14]. Desse modo, com o decorrer dos anos, as pessoas idosas tendem a valorizar as oportunidades de práticas religiosas a ponto de ressignificar sua existência conforme relatado pelo P74. Na perspectiva da teoria da Atividade, o idoso tende a engajar-se em atividades psicossociais, evitando a instalação da inatividade, sendo a religiosidade/espiritualidade um subsídio à preservação de vínculos sociais $[8,15]$. 
Houve ainda conteúdos referentes às influências do posicionamento religioso como componente capaz de justificar a busca pelos serviços de saúde, uma vez que a concepção de Deus identificada foi a de uma fonte primária de apoio, de conforto espiritual e para quem eles direcionam suas súplicas nos problemas pessoais e de saúde, fato corroborado por outras investigações [18-19]. O médico foi a autoridade a qual os idosos recorrem quando a doença persiste, gera dor ou limitação conforme expresso pelo P25.

As formas de comunicação/interação social são capazes de exercer diferentes níveis de influências sobre os comportamentos de idosos os quais compõem as crenças do tipo $D$ (Derivadas) [9-11]. Elas versaram sobre influência de: 1) meios de comunicação; 2) família; 3) meio social (extra)familiar; 4) serviços sociais/saúde.

Os conteúdos assumiram, na percepção dos participantes, características dicotômicas com valores positivos e negativos. O P12 descreveu a influência dos meios de comunicação sobre suas concepções e comportamentos, ao mencionar o uso da televisão para manter-se atualizado e obter informações aplicáveis ao seu cotidiano. A Teoria da Seletividade Socioemocional, ao abordar o declínio das interações sociais, justifica a busca por opções alternativas de distração para preencherem seu cotidiano $[8,13]$.

Os vínculos familiares foram considerados pelos idosos como influências positivas que qualificam sua convivência e thes possibilitam o recebimento de apoio e cuidados, conforme discurso do P14. A Teoria de Lawton no Constructo de Qualidade de Vida na Velhice descreve as possíveis influências familiares nesta fase da vida e das relações no contexto social [7,14].

Influências positivas advindas da convivência dos participantes com pessoas que não são familiares justificam a presença de vínculos de amizade, consideração e respeito, construídos ao longo dos tempos. Entre as crenças que versaram sobre a influência dos serviços sociais/de saúde foram identificadas aquelas que expressaram o recebimento de cuidados especializados providos pelos profissionais de saúde (P10). A assertiva anterior, ao ser analisada segundo a Teoria da Economia Política, explica como serviços sociais/de saúde agem no que tange ao acolhimento, ao tratamento e à forma de a pessoa idosa perceber seu atendimento $[8,15]$.

As crenças do tipo $E$ (inconsequentes) possuem baixa ligação com as demais presentes no sistema, uma vez que não possuem uma justificativa plausível para a sua existência, retratam apenas uma condição de gosto ou preferência $[7,8]$. Elas apresentaram conteúdos: 1) atividades laborais e; 2) serviços sociais/saúde.

O P19 retratou sua preferência por trabalhar, por manter-se ativo e ocupado. Fato explicado pela teoria da Atividade ao contemplar tarefas diárias idealizadas como necessidades de manutenção do engajamento em atividades psicossociais, de modo a prevenir a inatividade $[8,15]$.

Os serviços sociais/de saúde apresentaram crenças dicotômicas: na concepção positiva, foram associados a atendimentos anteriores, nos quais o idoso se sentiu acolhido, valorizado como o foco da atenção profissional; na dimensão negativa, o atendimento prestado pelos profissionais de saúde não esteve em consonância com a expectativa prévia dos participantes conforme relatos (P33 e P43).

O eixo imaginário de centralidade/perifericidade do sistema de crenças de Rokeach para o processo de envelhecimento tendeu à perifericidade com 696 crenças centrais (A e B) com os seguintes conteúdos: 1) caracterização do processo de agrisalhamento; 2) sentimento de ser útil; 3) inserção em atividades; 4) hábitos de vida; 5) limitações; 6) valores; 7) aprendizado de vida; 8) continuum saúde/doença; 9) avaliação de ser idoso; 10) finitude. Foram identificadas 1.169 crenças periféricas ( $C, D$ e E) cujos conteúdos foram: 1) Deus; 2) religiosidade/espiritualidade; 3) prática religiosa; 4) posicionamento religioso e busca pelos serviços de saúde; 5) meios de comunicação; 6) família; 7) meio social (extra)familiar; 8) serviços sociais/de saúde; 9) atividades laborais; 11) serviços sociais/de saúde, passíveis de serem acessadas/modificações.

Em síntese, estes resultados servem de alicerce para o raciocínio sobre a prática clínica de enfermeiros que atuam no contexto da APS a partir da compreensão do sistema de crenças e suas explicativas teóricas a respeito do processo de envelhecimento. O planejamento de ações/intervenções de enfermagem deve estar voltado para a modificação das crenças periféricas de caráter negativo, uma vez que estas superaram o número de crenças positivas. A premissa para a modificação das crenças periféricas negativas baseia-se em seu menor respaldo/consenso no grupo sendo, portanto, possíveis de serem influenciadas por abordagens interativas de socialização e/ou atividades de educação em saúde (prevenção, promoção e orientações sobre os cuidados em saúde numa concepção ampliada e multifatorial). 
A análise do sistema de crenças de pessoas com idade $\geq 65$ anos sobre o processo de envelhecimento, utilizando o referencial de crenças de Rokeach, permitiu identificar comportamentos, opiniões, informações, preferências, valores e concepções dos participantes a respeito das implicações sociais, econômicas, espirituais, das políticas públicas e de saúde sobre o envelhecer que, por vezes, foram negativas e de desvalorização dessa fase da vida.

Embora a extrapolação dos resultados seja uma limitação da investigação, é possível inferir sobre as concepções a respeito do processo do envelhecimento extraídas de fragmentos de discursos, as quais explicitam conteúdos que estão em consonância com as explicações teóricas sobre o envelhecimento humano podendo ser aproximado com resultados encontrados em futuras investigações em outros grupos sociais.

O fato de o eixo imaginário de centralidade/perifericidade do sistema de crenças ter tendido para a perifericidade aponta a possibilidade de a enfermagem utilizar os conteúdos periféricos emergentes advindos dos discursos dos participantes para estruturação de intervenções terapêuticas, que valorizem as pessoas em processo de envelhecimento, o que pode reverter concepções negativas sobre essa fase da vida.

\section{Referências}

1. Brasil. Projeções da população: Brasil e unidades da federação: revisão 2018. Coordenação de População e Indicadores Sociais. -2. ed. - Rio de Janeiro: IBGE, 2018. 58 p.

2. World Health Organization. Health statistics 2016: monitoring health for the SDGs sustainable development goals. World Health Organization, 2016. https://www.who.int/gho/publications/world health statistics/2016/en/

3. Arpino B, Solé-Auró A. Education inequalities in health among older European men and women: the role of active aging. J Aging Health 2017;31(1):185-208. https://doi.org/10.1177/ 0898264317726390

4. Uchomanowicz I, Jankowska-Polanska B, Wleklik M, Lisiak M, Gobbens R. Frailty syndrome: nursing interventions. SAGE Open Nursing 2018;4:1-11. https://doi.org/10.1177/2377960818759449

5. Teguo MT, Simo M, Lorenzo N, Rinaldo N, Cesari L, Matteo et al. Frailty syndrome among elderly in Caribbean region. Journal of the American Medical Directors Association 2017;18(6):547-48. https://doi.org/10.1016/j.jamda.2017.03.009

6. Chen C, Goldman DP, Zissimopoulos J, Rowe JW. Multidimensional comparison of countries' adaptation to societal aging. Proceedings of The National Academy of Sciences 2018;115(37):9169-74. https://doi.org/10.1073/pnas.1806260115

7. Martins R. Aging, retrogenesis of motor development, physical exercise and health promotion. Portuguese Society of Physical Education Bulletin 2017;(32);31-40.

8. Techera MP, Ferreira AH, Sosa CL, Marco NV, Muñoz LA. Significados que le atribuyen al envejecimiento activo y saludable un grupo de personas mayores que viven en comunidad. Texto Contexto Enferm 2017;26(3):1-9. https://doi.org/10.1590/0104-07072017001750016

9. Rokeach M. Understanding human values. Free Press: NY; 2008.

10. Bardin L. Análise de conteúdo. Lisboa: Edições 70; 2016. p. 280.

11. Pelinka A. Attitude change: the competing views. New York: Routledge: NY; p.259.

12. Barcelos AMF. As influências das crenças dos alunos acerca do corpo docente em relação à aprendizagem de Línguas. Rev Brasileira de Linguística Aplicada 2007;7(2):109-38.

13. Martin S, Lipsky MD, King MDM. Biological theories of aging. Disease-a-Month 2015;61(11):460-6. https://doi.org/10.1016/j.disamonth.2015.09.005

14. Wernher MSI, Martin S, Lipsky MD. Psychological theories of aging. Disease-a-Month 2015;61(11):480-88.

15. Hasworth SB, Cannon ML. Social theories of aging. Disease-a-Month 2015;61(11):47579. https://doi.org/10.1016/j.disamonth.2015.09.003

16. Melo LD. O Processo de envelhecimento para pessoas idosas: estudo de representações sociais e crenças de Rokeach [Dissertação]. Faculdade de Enfermagem da Universidade Federal de Juiz de Fora; 2015. p.189. [citado 2020 Jan 
10]. Disponível em:

https://bdtd.ibict.br/vufind/Record/UFJF c33c84b95b80963fb4dad26ecd6ffb17

17. Dias JA, Arreguy-Sena C, Pinto PF, Souza LCD. Ser idoso e o processo do envelhecimento: saúde percebida. Escola Anna Nery 2011;15(2):372-9.

https://doi.org/10.1590/S1414-81452011000200021

18. Lifshitz R, Nimrod G, Bachener YG. Spirituality and wellbeing in later life: a multidimensional approach. Aging e Mental Health 2019;23(8):984-91.

https://doi.org/10.1080/13607863.2018.1460743

19. Melo-Tavares M, Gomes AMT, Barbosa DJ, Rocha JCCC, Bernardes MMR, SilvaThiengo PC. Spirituality and religiosity in the daily routine of hospital nursing. Journal of Nursing UFPE on line 2018;12(4):1097-102. https://doi.org/10.5205/1981-8963v12i4a234371p129-139-2018 